\title{
IS THE CRITICAL PERCOLATION PROBABILITY LOCAL?
}

\author{
ITAI BENJAMINI, ASAF NACHMIAS AND YUVAL PERES
}

\begin{abstract}
We show that the critical probability for percolation on a $d$-regular nonamenable graph of large girth is close to the critical probability for percolation on an infinite $d$-regular tree. This is a special case of a conjecture due to O. Schramm on the locality of $p_{c}$. We also prove a finite analogue of the conjecture for expander graphs.
\end{abstract}

\section{INTRODUCTION}

Denote by $p_{c}(G)$ the critical probability for Bernoulli bond percolation on an infinite graph $G$, that is,

$$
p_{c}(G)=\inf \left\{p \in[0,1]: \mathbf{P}_{p}(\exists \text { an infinite component })>0\right\} .
$$

Is the value of $p_{c}$ determined by the local geometry of the graph or by global properties (such as volume growth and expansion)? In this note we show that the former is the correct answer for non-amenable graphs with tree-like local geometry, and discuss a conjecture of Schramm that $p_{c}$ is locally determined in greater generality.

Recall that the girth $g$ of a graph $G$ is the minimum length of a cycle in $G$. Let $P$ be the transition matrix of the simple random walk (SRW) on $G$ and let $I$ be the identity matrix. The bottom of the spectrum of $I-P$ is defined to be the largest constant $\lambda_{1}$ with the property that for all $f \in \ell^{2}(G)$ we have

$$
\langle f,(I-P) f\rangle \geq \lambda_{1}\langle f, f\rangle .
$$

Kesten ([9], [10]) proved that $G$ is a non-amenable Cayley graph if and only if $\lambda_{1}>0$. This was extended by Dodziuk [7] to general infinite bounded degree graphs (for more background on non-amenability see [11] and [14]).

Theorem 1.1. There exists an absolute constant $C>0$ such that if $G$ is a nonamenable regular graph with degree $d$ and girth $g$ such that the bottom of spectrum of $I-P$ is $\lambda_{1}>0$, then

$$
p_{c}(G) \leq \frac{1}{d-1}+\frac{C \log \left(1+\frac{1}{\lambda_{1}^{2}}\right)}{d g} .
$$


Recall that $p_{c}\left(T_{d}\right)=\frac{1}{d-1}$ where $T_{d}$ is an infinite $d$-regular tree and that for any $d$ regular graph $G$ we have $p_{c}(G) \geq \frac{1}{d-1}$. Thus, Theorem 1.1 asserts that non-amenable graphs with large girth and degree $d$ have $p_{c}$ close to the lowest possible value, $p_{c}\left(T_{d}\right)$.

It is easy to construct non-amenable graphs with arbitrary girth, for example, take the Cayley graph of $\left\langle a, b, c \mid c^{n}=1\right\rangle$. Olshanskii and Sapir [13] constructed for any $k \geq 2$ a group $\Gamma_{k}$ with the following property. For any $\ell>0$ there is a set $S_{\ell}$ consisting of $k$ generators for $\Gamma_{k}$, such that the Cayley graph $G\left(\Gamma_{k}, S_{\ell}\right)$ has girth at least $\ell$ and $\inf _{\ell} \lambda_{1}\left(G\left(\Gamma_{k}, S_{\ell}\right)\right)>0$ (as remarked in [13], for $k \geq 4$ such groups were also constructed by Akhmedov [2]).

Let $G$ be a graph and $v$ a vertex in $G$. Denote by $B_{G}(v, R)$ the ball of radius $R$ in $G$ centered at $v$, in the graph metric, with its induced graph structure. We say that a sequence of transitive graphs $G_{n}$ converges to $G$ if for any integer $R>0$ there exists $N$ such that $B_{G_{n}}\left(v_{n}, R\right)$ and $B_{G}(v, R)$ are isomorphic as rooted graphs, for all $n \geq N$ (note that the choices of $v_{n}$ and $v$ are irrelevant due to transitivity). Oded Schramm (personal communication) suggested the following conjecture.

Conjecture 1.2. Let $G_{n}$ be sequence of vertex transitive infinite graphs with $\sup _{n} p_{c}\left(G_{n}\right)<$ 1 such that $G_{n}$ converges to a graph $G$. Then $p_{c}\left(G_{n}\right) \rightarrow p_{c}(G)$.

This conjecture is open for infinite graphs even if we assume that they are uniformly nonamenable. We can prove the following analogue of the conjecture for finite expander graphs, by extending the analysis of [1], Proposition 3.1. For each $n \geq 1$, let $G_{n}$ be a finite graph and let $U_{n}$ be a uniformly chosen random vertex in $G_{n}$. We say that the sequence of finite graphs $\left\{G_{n}\right\}$ converges weakly to an infinite rooted graph $(G, \rho)$ (where $\rho$ is a fixed vertex of $G$ ) if for each $R>0$ we have

$$
\mathbf{P}\left(B_{G_{n}}\left(U_{n}, R\right) \neq B_{G}(\rho, R)\right) \rightarrow 0 \quad \text { as } n \rightarrow \infty
$$

where the event above means that the balls are not isomorphic as rooted graphs. This is a special case of the graph limits defined in [5]. For two sets of vertices $A$ and $B$, write $E(A, B)$ for the set of edges with one endpoint in $A$ and the other in $B$. Recall that the Cheeger constant $h(G)$ of a finite graph $G=(V, E)$ is defined by

$$
h(G)=\min _{A \subset V}\left\{\frac{|E(A, V \backslash A)|}{|A|}: 0<|A| \leq|V| / 2\right\} .
$$

Theorem 1.3. Let $(G, \rho)$ be an infinite bounded degree rooted graph and let $G_{n}$ be a sequence of finite graphs with uniform Cheeger constant $h>0$ and a uniform degree bound $d$, such that $G_{n} \rightarrow G$ weakly. Let $p \in[0,1]$ and write $G_{n}(p)$ for the graph of open 
edges obtained from $G_{n}$ by performing bond percolation with parameter $p$. If $p<p_{c}(G)$, then for any constant $\alpha>0$ we have

$$
\mathbf{P}\left(G_{n}(p) \text { contains a component of size at least } \alpha\left|G_{n}\right|\right) \rightarrow 0 \text { as } n \rightarrow \infty,
$$

and if $p>p_{c}(G)$, then there exists some $\alpha>0$ such that

$$
\mathbf{P}\left(G_{n}(p) \text { contains a component of size at least } \alpha\left|G_{n}\right|\right) \rightarrow 1 \text { as } n \rightarrow \infty \text {. }
$$

For the reader's convenience, we present the proof of Theorem 1.3 in Section 4 .

1.1. Further discussion. Conjecture 1.2 suggests that the critical percolation probability is locally determined. This contrasts with critical exponents which are believed to be universal and depend only on global properties of the graph. For instance, the value of $p_{c}$ on the the two dimensional square lattice is $\frac{1}{2}$, but on the two dimensional triangular lattice it is $2 \sin (\pi / 18)$; however, the critical exponents are believed to be the same.

It is worth noting another example of the locality of $p_{c}$ where the limit graph is the lattice $\mathbb{Z}^{d}$. For $d>1$ and $n>1$, write $\mathbb{Z}_{n}^{d}$ for the $d$-dimensional torus with side $n$. The following theorem is an immediate corollary of a theorem of Grimmett and Marstrand [8] combined with the fact that the critical probability of a quotient graph is always at least the critical probability of the original graph (see [4], 6] or [11]).

Theorem 1.4 (Grimmett, Marstrand [8]). For any $d>1$ and $k$ satisfying $2 \leq k<d$ we have

$$
p_{c}\left(\mathbb{Z}^{k} \times \mathbb{Z}_{n}^{d-k}\right) \rightarrow p_{c}\left(\mathbb{Z}^{d}\right) \quad \text { as } n \rightarrow \infty \text {. }
$$

Observe that this is theorem is a special case of Conjecture 1.2. For background and further conjectures regarding percolation on infinite graphs see [4, 11].

\section{UNIFORM ESCAPE PROBABILITY}

In this section we prove a useful lemma.

Lemma 2.1. Consider a reversible irreducible Markov chain $\left\{X_{t}\right\}$ on a countable state space $V$, with infinite stationary measure $\pi$ and transition matrix $P$, such that the bottom of the spectrum of $I-P$ is $\lambda_{1}>0$ (that is, (1.1) holds for any $f \in \ell^{2}(\pi)$ ). Let $A \subset V$ be a nonempty set of states with $\pi(A)<\infty$ and let $\pi_{A}(\cdot)=\pi(\cdot) / \pi(A)$ be the normalized restriction of $\pi$ to $A$. Then

$$
\mathbf{P}_{\pi_{A}}\left(X_{t} \text { never returns to } A\right) \geq \lambda_{1} \text {. }
$$


Proof. Let $B \subset V$ be disjoint from $A$ such that $V \backslash(A \cup B)$ is finite. Define

$$
\tau=\min \left\{t \geq 0: X_{t} \in A \cup B\right\} \quad \text { and } \quad \tau^{+}=\min \left\{t>0: X_{t} \in A \cup B\right\} .
$$

The irreducibility assumption and the finiteness of the complement of $A \cup B$ imply that $\tau^{+}<\infty$ a.s. for any starting state. We will show that for all sets $B$ as above,

$$
\mathbf{P}_{\pi_{A}}\left(X_{\tau^{+}} \in B\right) \geq \lambda_{1}
$$

The assertion of the lemma then follows by enumerating $V \backslash A$ as $v_{1}, v_{2}, v_{3}, \ldots$, taking $B=B_{k}=\left\{v_{j}: j \geq k\right\}$ and intersecting the events in (2.1) over all these sets $B=B_{k}$ for $k \geq 1$. Let

$$
f(x)=\mathbf{P}_{x}\left(X_{\tau} \in A\right) .
$$

Observe that $f \equiv 1$ on $A$ and $f \equiv 0$ on $B$. For all $x \in G$,

$$
(P f)(x)=\mathbf{P}_{x}\left(X_{\tau^{+}} \in A\right) .
$$

In particular, $f$ is harmonic (satisfies $P f=f)$ on $G \backslash(A \cup B)$. Thus $((I-P) f)(x)=$ $\mathbf{P}_{x}\left(X_{\tau^{+}} \in B\right)$ for $x \in A$ and $((I-P) f)(x)=0$ for $x \in G \backslash(A \cup B)$. Therefore

$$
\langle f,(I-P) f\rangle=\sum_{x \in A} \pi(x) \mathbf{P}_{x}\left(X_{\tau^{+}} \in B\right)=\pi(A) \mathbf{P}_{\pi_{A}}\left(X_{\tau^{+}} \in B\right) .
$$

On the other hand, clearly,

$$
\langle f, f\rangle \geq \sum_{x \in A} \pi(x) f(x)^{2}=\pi(A) .
$$

The claim (2.1) follows by inserting the last two formulas in (1.1).

\section{Proof of Theorem 1.1}

We return to the setting of Theorem 1.1. Let $G$ be regular graph of degree $d$ and girth $g$ and write $\widetilde{g}:=\lceil g / 2\rceil-1$. Given a set of vertices $A$ in $G$ and $\alpha \in(0,1)$, we say that an edge $(x, u)$ is $(\alpha, A)$-good if $x \in A$ and at least an $\alpha$ fraction of the $(d-1)^{\widetilde{g}}$ non-backtracking paths of length $\widetilde{g}$ emanating from $u$, for which the first step is not $x$, avoid $A$ (in particular, $u \notin A$.) The following lemma is a corollary of Lemma 2.1 ,

Corollary 3.1. Let $G$ be a regular graph with degree $d$ and girth $g$. If $G$ is nonamenable, i.e., it satisfies $\lambda_{1}>0$, then for any finite set $A \subset V(G)$, there exist at least $\frac{\lambda_{1} d}{2}|A|$ edges $(x, u)$ which are $\left(\lambda_{1} / 2, A\right)$-good. 
Proof. For an edge $(x, u)$ with $x \in A$, let $\beta_{(x, u)}=\mathbf{P}_{x}\left(X_{1}=u\right.$ and $\left.\forall t>0 \quad X_{t} \notin A\right)$, where $\left\{X_{t}\right\}$ is a SRW in $G$, started at $x$. Let $\tau:=\min \left\{t: \operatorname{dist}\left(u, X_{t}\right)=\widetilde{g}\right\}$. Since the ball $B_{G}(u, \widetilde{g})$ is a spherically symmetric tree, the loop erasure of $\left(X_{t}\right)_{t=0}^{\tau}$ yields a uniform random non-backtracking path of length $\widetilde{g}$ from $u$. Thus if $\beta_{(x, u)} \geq \alpha$, then the edge $(x, u)$ is $(\alpha, A)$-good. By Lemma 2.1,

$$
\frac{1}{d|A|} \sum_{(x, u): x \in A} \beta_{(x, u)} \geq \lambda_{1},
$$

and we conclude that at least $\frac{\lambda_{1} d}{2}|A|$ edges $(x, u)$ with $x \in A$ must satisfy $\beta_{(x, u)} \geq$ $\lambda_{1} / 2$.

Proof of Theorem 1.1. Let $\epsilon>0$ be a small number and set $p=\frac{1}{d-1}+\epsilon$. For each edge $e$ we draw two independent Bernoulli random variables $X_{e}(p)$ and $Y_{e}(\epsilon)$ with means $p$ and $\epsilon$ respectively. We say that an edge is open if one of these variables takes the value 1 and closed otherwise. We also say that the edge $e$ is $p$-open if $X_{e}(p)=1$ and $\epsilon$-open if $Y_{e}(\epsilon)=1$. For a vertex $v$ we write $\mathcal{C}(v)$ for the open cluster of $v$. The probability that an edge is closed is $(1-p)(1-\epsilon)$, hence $|\mathcal{C}(v)|$ is dominated by the cluster size in $(p+\epsilon)$-bond percolation. Our goal is to show that with positive probability $|\mathcal{C}(v)|=\infty$.

We perform the following exploration process, which will produce an increasing sequence $\left\{A_{t}\right\}$ of connected vertex sets in which $A_{t} \subset \mathcal{C}(v)$ for all $t$. At each step, some of the edges touching $A_{t}$ will be $\epsilon$-closed and some will be $\epsilon$-unchecked. We begin by setting $A_{0}$ to be the $p$-cluster of $v$ (that is, all the vertices connected to $v$ by $p$-open paths) and all the edges touching $A_{0}$ are $\epsilon$-unchecked. We assume that $A_{0}$ is finite (otherwise we are finished). At step $t>1$ let $\mathcal{E}_{t-1}$ be the set of $\epsilon$-unchecked edges $(x, u)$ such that $(x, u)$ is $\left(\lambda_{1} / 2, A_{t-1}\right)$-good. If $\mathcal{E}_{t-1}$ is empty, the process ends. If not, we choose $(x, u) \in \mathcal{E}_{t-1}$ according to some prescribed ordering of the edges and check whether the edge is $\epsilon$-open. If it is $\epsilon$-closed we put $A_{t}=A_{t-1}$ and continue to the next step of the process. Otherwise, we let

$$
A_{t}=A_{t-1} \cup \mathcal{V}
$$

where $\mathcal{V}$ is the set of vertices $v$ of distance at most $\widetilde{g}$ from $u$ such that the unique path of length at most $\widetilde{g}$ between $u$ and $v$ avoids $A_{t-1}$ and is $p$-open.

This finishes the description of the exploration process. To analyze this process we introduce the following random variable

$$
Z_{t}=\mid\left\{e: e \text { is an } \epsilon \text {-closed and } \epsilon \text {-checked edge touching } A_{t}\right\} \mid .
$$


Let $\tau$ be the stopping time

$$
\tau=\min \left\{t:\left|A_{t}\right|<\frac{2 t}{\lambda_{1} d}\right\}
$$

At each step we check the $\epsilon$-status precisely one edge, hence $Z_{t} \leq t$ for all $t$. Thus, by Corollary 3.1, if $\left|A_{t}\right|>\frac{2 t}{\lambda_{1} d}$ there must exist at least one $\epsilon$-unchecked edge $(x, u)$ which is $\left(\lambda_{1} / 2, A_{t}\right)$-good. Write $\mathcal{F}_{t}$ for the $\sigma$-algebra generated by the $\epsilon$ and $p$ status of the edges we examined in the exploration process up to time $t$ and let $\xi_{t}=\left|A_{t+1}\right|-\left|A_{t}\right|$. By the discussion above we have that

$$
\mathbb{E}\left[\xi_{t} \mid \mathcal{F}_{t-1}, \tau>t\right] \geq \frac{\epsilon \lambda_{1}}{2} \sum_{j=1}^{\widetilde{g}}(1+\epsilon(d-1))^{j} \geq \frac{\lambda_{1}\left[(1+\epsilon(d-1))^{\widetilde{g}}-1\right]}{2(d-1)} .
$$

To see the first inequality in (3.1), recall that $(x, u)$ is $\epsilon$-open with probability $\epsilon$. Also, for any $j \leq \widetilde{g}$ the expected number of vertices of distance $j$ from $u$ such that the path between them and $u$ avoids $A_{t}$ and is $p$-open is at least $\frac{\lambda_{1}}{2}(p(d-1))^{j}=\frac{\lambda_{1}(1+\epsilon(d-1))^{j}}{2}$.

We now assume that

$$
\widetilde{g} \geq \frac{\log \left(1+\frac{8}{\lambda_{1}^{2}}\right)}{\log (1+\epsilon(d-1))}
$$

so that $\mathbb{E}\left[\xi_{t} \mid \mathcal{F}_{t-1}, \tau>t\right] \geq 4 d^{-1} \lambda_{1}^{-1}$ by (3.1). Since $\left|\xi_{t}\right| \leq(d-1)^{\widetilde{g}}$, Azuma-Hoeffding's inequality (see Chapter 7 of [3]) gives that for any $t>1$

$$
\mathbf{P}\left(\tau=t+1 \mid A_{0}\right) \leq \mathbf{P}\left(\sum_{i=1}^{t} \xi_{i} \leq \frac{2 t}{d \lambda_{1}}\right) \leq e^{-c t},
$$

where $c=2 \lambda_{1}^{-2} d^{-2}(d-1)^{-2 \widetilde{g}}>0$. Since $\left|A_{t}\right|$ is a non-decreasing sequence we have that $\tau>\frac{\lambda_{1} d\left|A_{0}\right|}{2}$. For any $K>0$ there is some positive probability (depending on $K$ ) of having $\left|A_{0}\right| \geq K$ and we infer from (3.3) that

$$
\begin{aligned}
\mathbf{P}(\tau=\infty) & \geq \mathbf{P}\left(\left|A_{0}\right| \geq K\right) \mathbf{P}\left(\tau=\infty|| A_{0} \mid \geq K\right) \\
& \geq \mathbf{P}\left(\left|A_{0}\right| \geq K\right)\left[1-\sum_{t \geq \frac{\lambda_{1} d K}{2}} e^{-c t}\right]>0,
\end{aligned}
$$

as long as we choose $K=K\left(g, \lambda_{1}, d\right)$ to be large enough. The event $\tau=\infty$ implies that $|\mathcal{C}(v)|=\infty$, and hence, by (3.2) when $\epsilon \geq C(d g)^{-1} \log \left(1+\frac{1}{\lambda_{1}^{2}}\right)$ there is positive probability of an infinite component in $\left(\frac{1}{d-1}+2 \epsilon\right)$-bond percolation (for $\epsilon \leq(d-1)^{-1}$ one can take $C=128$ using the inequalities $\log (1+8 x) \leq 8 \log (1+x)$ and $\log (1+x) \geq$ $x / 2$ valid for $x \in(0,1))$. This concludes the proof of the theorem. 


\section{Proof of Theorem 1.3}

Without loss of generality assume that $\left|G_{n}\right|=n$. We first take $p<p_{c}(G)$ and fix $\alpha>0$. Since $p<p_{c}(G)$, for any $\epsilon>0$ there exists $R=R(\epsilon)$ large enough such that in G

$$
\mathbf{P}_{p}(\rho \leftrightarrow \partial B(\rho, R))<\epsilon .
$$

Thus for large enough $n$ we have in $G_{n}$

$$
\mathcal{L} \times \mathbf{P}_{p}\left(U_{n} \leftrightarrow \partial B\left(U_{n}, R\right)\right) \leq \epsilon,
$$

where $\mathcal{L}$ is the law of $U_{n}$. Since $G$ has bounded degree, we deduce that for any $\epsilon>0$ there exists $n$ large enough such that

$$
\mathcal{L} \times \mathbf{P}_{p}\left(\left|\mathcal{C}\left(U_{n}\right)\right| \geq d^{R+1}\right) \leq \epsilon .
$$

Write $\mathcal{C}_{1}(n)$ for the largest component of $G_{n}(p)$ and note that as long as $d^{R+1} \leq \alpha n$ we have that

$$
\mathcal{L} \times \mathbf{P}_{p}\left(\left|\mathcal{C}\left(U_{n}\right)\right| \geq d^{R+1}\right) \geq \alpha \mathbf{P}_{p}\left(\left|\mathcal{C}_{1}(n)\right| \geq \alpha n\right)
$$

and we get that

$$
\mathbf{P}_{p}\left(\left|\mathcal{C}_{1}(n)\right| \geq \alpha n\right) \leq \epsilon \alpha^{-1}
$$

which proves the first assertion of the theorem.

To prove the second assertion of the theorem we use a sprinkling argument, as in [1]. Assume $p>p_{c}(G)$ and for some $\epsilon>0$ let $p_{1}=p_{c}(G)+\epsilon$ such that $1-p=\left(1-p_{1}\right)(1-\epsilon)$. We first consider $G_{n}\left(p_{1}\right)$. Since $p_{1}>p_{c}(G)$, there exists some $\delta>0$ such that for all $R>0$ we have

$$
\mathbf{P}_{p_{1}}(\rho \leftrightarrow \partial B(\rho, R)) \geq \delta .
$$

For $v \in G_{n}$, write $B_{p_{1}}(v, R)$ for the set of vertices in $G_{n}\left(p_{1}\right)$ which are connected to $v$ in a $p_{1}$-open path of length at most $R$. We get that for any $R>0$ there exists $n_{0}$ such that for $n \geq n_{0}$ we have in $G$

$$
\mathcal{L} \times \mathbf{P}\left(\left|B_{p_{1}}\left(U_{n}, R\right)\right| \geq R\right) \geq \delta / 2 .
$$

Let $X_{R}$ denote the random variable

$$
X_{R}=\left|\left\{v \in G_{n}:\left|B_{p_{1}}(v, R)\right| \geq R\right\}\right|,
$$


so that $\mathbb{E} X_{R} \geq \delta n / 2$. On the other hand, note that changing the status of a single edge can change $X_{R}$ by at most $d^{R}$, where $d$ is the degree bound of $G_{n}$. The method of bounded differences (see Theorem 3.1 of [12] or Chapter 7 of [3]) gives that

$$
\mathbf{P}\left(X_{R} \leq \frac{\delta n}{4}\right) \leq \exp \left(-\frac{\delta^{2} n}{8 d^{2 R}}\right) .
$$

Assume now that $X_{R} \geq \delta n / 4$ and consider the connected components of $G_{n}\left(p_{1}\right)$ of size at least $R$. Their number $m$ is at most $n / R$. We now consider the union of $G_{n}\left(p_{1}\right)$ with $G_{n}(\epsilon)$ and claim that many of these components join together by edges of $G_{n}(\epsilon)$ and create a component of linear size. Indeed, consider a partitition of the $m$ large components of $G_{n}\left(p_{1}\right)$ into two sets, $A$ and $B$, each spanning at least $\delta n / 12$ vertices. If for any such partition there is an open path in $G_{n}(\epsilon)$ connecting $A$ and $B$, then there exists a component of size at least $\delta n / 12$ in $G_{n}\left(p_{1}\right) \cup G_{n}(\epsilon)$. Since $G_{n}$ has Cheeger constant at least $h>0$ we get by Menger's Theorem that for any such $A$ and $B$ there are at least $h \delta n / 12$ edge disjoint paths connecting $A$ to $B$. Since there are most $d n / 2$ edges in $G_{n}$ we have that at least a half of these paths must be of length at most $\frac{12 d}{h \delta}$. The probability that all these paths are closed in $G_{n}(\epsilon)$ is at most

$$
\left[1-\epsilon^{\frac{12 d}{h \delta}}\right]^{\frac{h \delta n}{24}} \text {. }
$$

There are at most $2^{m}$ different possibilities for choosing $A$ and $B$. Hence, the probability that there exists such $A$ and $B$ is at most

$$
2^{m}\left[1-\epsilon^{\frac{12 d}{h \delta}}\right]^{\frac{h \delta n}{24}} \leq \exp \left(n / R-\epsilon^{\frac{12 d}{h \delta}} h \delta n / 24\right),
$$

which goes to 0 as long as $R$ is chosen such that $R^{-1}<\epsilon^{\frac{12 d}{h \delta}} h \delta$. This together with (4.1) shows that

$$
\mathbf{P}\left(G_{n}(p) \text { contains a component of size at least } \frac{\delta n}{12}\right) \rightarrow 1 \quad \text { as } n \rightarrow \infty .
$$

Acknowledgements: We are indebted to Mark Sapir and Oded Schramm for useful discussions.

\section{REFERENCES}

[1] N. Alon, I. Benjamini and A. Stacey, Percolation on finite graphs and isoperimetric inequalities Ann. Probab. 32 (2004), 1727-1745.

[2] A. Akhmedov, The girth of groups satisfying Tits Alternative, J. of Algebra, 287 (2005), no.2, 275-282. 
[3] N. Alon and J. H. Spencer, The probabilistic method, 2nd edition, Wiley, New York, 2000.

[4] I. Benjamini and O. Schramm, Percolation Beyond $Z^{d}$, Many Questions And a Few Answers, ECP, 1 (1996), Paper no. 8.

[5] I. Benjamini and O. Schramm, Recurrence of Distributional Limits of Finite Planar Graphs, Electron. J. Probab. Vol. 6, no. 23, 13 pp.

[6] M. Campanino, Inequalities for critical probabilities in percolation, Particle Systems, Random Media and Large Deviations, volume 41 of Contemp. Math., pages 1-9, R. Durrett editor. Amer. Math. Soc., Providence, RI. Proceedings of the AMSIMS- SIAM joint summer research conference in the mathematical sciences on mathematics of phase transitions held at Bowdoin College, Brunswick, Maine, June 24-30, (1984).

[7] J. Dodziuk, Difference equations, isoperimetric inequality and transience of certain random walks, Trans. Amer. Math. Soc., 284, 787-794.

[8] G. R. Grimmett and J. M. Marstrand, The supercritical phase of percolation is well behaved, Proc. Roy. Soc. London Ser. A 430 (1990), no. 1879, 439-457.

[9] H. Kesten, Full Banach mean values on countable groups, Math. Scand., 7, 146-156.

[10] H. Kesten, Symmetric random walks on groups, Trans. Amer. Math. Soc., 92, 336-354.

[11] R. Lyons with Y. Peres, Probability on Trees and Networks, In preparation, http://mypage.iu.edu/ rdlyons/prbtree/prbtree.html

[12] C. McDiarmid, Concentration. Probabilistic methods for algorithmic discrete mathematics, 195-248, Algorithms Combin., 16, Springer, Berlin, (1998).

[13] A. Yu. Olshanskii and M. V. Sapir, On $k$-free-like groups, preprint. Available at http://arxiv.org/abs/0811.1607

[14] W. Woess, Random walks on infinite graphs and groups, Cambridge Tracts in Mathematics, 138, Cambridge University Press, Cambridge.

Itai Benjamini: itai.benjamini (at)weizmann.ac.il

The Weizmann Institute of Science,

Rehovot POB 76100, Israel.

Asaf Nachmias: asafn(at)microsoft.com

Microsoft Research, One Microsoft way,

Redmond, WA 98052-6399, USA.

Yuval Peres: peres(at)microsoft.com

Microsoft Research, One Microsoft way,

Redmond, WA 98052-6399, USA. 